PROCEEDINGS OF THE

AMERICAN MATHEMATICAL SOCIETY

Volume 125, Number 12, December 1997, Pages 3675-3677

S 0002-9939(97)04019-7

\title{
PRODUCTS OF POSITIVE OPERATORS
}

\author{
GERARD J. MURPHY
}

(Communicated by Palle E. T. Jorgensen)

\begin{abstract}
A new, very simple proof is given of a result of P. Y. Wu which asserts that every unitary operator on an infinite-dimensional Hilbert space is a product of positive operators.
\end{abstract}

A number of mathematicians have considered the problem of writing an operator as a product of "nice" operators, such as positive, hermitian or normal operators. Our principal reference for this is a paper of $\mathrm{P}$. Y. Wu [6], but see also [2] and [5]. This kind of question, and related questions, have also been considered in a $C^{*}$-algebra context, see [3].

A core result of Wu's paper is his theorem that a unitary operator on an infinitedimensional Hilbert space is a product of (sixteen) positive operators. This is an unexpected result, given what occurs in finite dimensions. For, in the latter situation, if we make the usual identification of an operator with a finite square matrix, a theorem of C. S. Ballantine [1] asserts that a matrix is a product of positive matrices precisely when its determinant is non-negative.

The aim of this paper is to present a new proof of Wu's unitary result that is simpler and more perspicuous. Our version is so simple that we do not even have to use Ballantine's theorem (Wu's proof of his unitary result does make use of this theorem). In fact, we derive a weak version of Ballantine's theorem from our methods that has the merit of also being very simple and perspicuous.

1. Lemma. Let $u$ be a unitary element of a unital $C^{*}$-algebra $A$. Then the matrix

$$
a=\left(\begin{array}{cc}
u & 0 \\
0 & u^{*}
\end{array}\right)
$$

is a product of eight positive elements of $M_{2}(A)$.

Proof. To see this, we need only show that the scalar matrix

$$
b=\left(\begin{array}{cc}
0 & -1 \\
1 & 0
\end{array}\right)
$$

is the product of four positive scalar matrices. For, in this case, using the fact that the matrix

$$
c=\left(\begin{array}{ll}
0 & 1 \\
u & 0
\end{array}\right)
$$

Received by the editors March 29, 1996 and, in revised form, July 22, 1996. 1991 Mathematics Subject Classification. Primary 46L05, 47A65.

(C) 1997 American Mathematical Society 
is a unitary in $M_{2}(A)$ and that $a=b\left(c b c^{*}\right)$, we conclude that $a$ is the product of eight positive elements of $M_{2}(A)$. To prove the positive factorisation for $b$, note first that

$$
b=\left(\begin{array}{cc}
1 / 2 & 0 \\
3 / 2 & 1
\end{array}\right)\left(\begin{array}{cc}
0 & -2 \\
1 & 3
\end{array}\right) .
$$

Thus, $b$ is the product of two matrices, each of which has distinct positive eigenvalues. It therefore suffices to show that any such matrix in $M_{2}(\mathbf{C})$ is the product of two positive matrices. Clearly, such a matrix is similar to a diagonal positive matrix $p$, so it is of the form $v p v^{-1}$, for some invertible matrix $v$. If $v=u q$ is the polar decomposition of $v$, then $v p v^{-1}=\left(u q p q u^{*}\right)\left(u q^{-2} u^{*}\right)$, so $v p v^{-1}$ is the product of two positive matrices, as required.

2. Lemma. Let $\left(u_{n}\right)$ be a sequence of unitaries on a Hilbert space $K$. Then the diagonal unitary $u$, having diagonal entries $u_{1}, u_{1}^{*}, u_{2}, u_{2}^{*}, \ldots$, on the Hilbert space direct sum $H=\bigoplus_{n=1}^{\infty} H_{n}$, where $H_{n}=K$ for all $n$, is a product of eight positive operators on $H$.

Proof. It follows from the proof of the preceding lemma that there exist four positive operators $p_{1}, p_{2}, p_{3}, p_{4}$ and, for each $n$, a unitary $v_{n}$ on $K \oplus K$ such that $u_{n} \oplus u_{n}^{*}=$ $p_{1} p_{2} p_{3} p_{4} v_{n} p_{1} p_{2} p_{3} p_{4} v_{n}^{*}$ (the operators $p_{1}, p_{2}, p_{3}, p_{4}$ do not depend on $n$ - they are just four positive factors of the matrix $b$ that occurs in the proof of the preceding lemma). Now form operator direct sums $P_{1}, P_{2}, P_{3}, P_{4}$ on $H=(K \oplus K) \oplus(K \oplus K) \oplus$ $\cdots$ in such a way that $P_{j}$ has constant diagonal entries $p_{j}$. Clearly, $P_{1}, P_{2}, P_{3}, P_{4}$ are positive operators and we have $u=P_{1} P_{2} P_{3} P_{4} V P_{1} P_{2} P_{3} P_{4} V^{*}$, where $V$ is the unitary $\bigoplus_{n} v_{n}$. Thus, $u$ is the product of eight positive operators on $H$.

3. Theorem (P. Y. Wu). Let $u$ be a unitary operator on an infinite-dimensional Hilbert space $H$. Then $u$ is the product of sixteen positive operators on $H$.

Proof. It follows from the spectral theorem that a unitary operator on an infinitedimensional Hilbert space is a direct sum of unitary operators on a sequence of equidimensional Hilbert subspaces. Using this, we may reduce to the case where there is a Hilbert space $K$ and unitary operators $u_{n}$ on $K$ such that $H$ is the Hilbert space direct sum $\bigoplus_{n=1}^{\infty} H_{n}$, with $H_{n}=K$ for all $n$, and $u=\bigoplus_{n} u_{n}$. Define unitaries $v_{n}$ on $K$ by induction as follows: $v_{1}=u_{1}, v_{2}=u_{1}^{*}, v_{2 n}=v_{2 n-1}^{*}$ and $v_{2 n+1}=u_{2 n+1} v_{2 n}^{*} u_{2 n}$. Then set $w_{n}=v_{n}^{*} u_{n}$ for all $n$. Denote by $v$ and $w$ the direct sums of the sequences $\left(v_{n}\right)$ and $\left(w_{n}\right)$, respectively. Clearly, $u=v w$. Also, since $v$ is a direct sum of a sequence of operators of the form $v_{1}, v_{1}^{*}, v_{3}, v_{3}^{*}, \ldots$ and $w$ is a direct sum of operators of the form $1, w_{2}, w_{2}^{*}, w_{4}, w_{4}^{*}, \ldots$, it follows from the preceding lemma that each of $v$ and $w$ is the product of eight positive operators on $H$.

We note that Wu's result was improved by N. C. Phillips-he shows that every unitary on an infinite-dimensional Hilbert space is a product of six positive operators [4]. However, our techniques do not appear to be sufficient to obtain this stronger result.

We now use Lemma 1 to give a quick proof of (a weak form of) Ballantine's theorem.

4. Theorem (C. S. Ballantine). An invertible matrix a in $M_{n}(\mathbf{C})$ is a product of positive invertible matrices if and only if its determinant is positive. 
Proof. The forward implication is trivial. Suppose then $a$ has positive determinant. If we polar decompose $a$, so that $a=u p$, with $u$ a unitary and $p=\left(a^{*} a\right)^{1 / 2}$, then $\operatorname{det}(u)>0$ and therefore $\operatorname{det}(u)=1$. To prove the theorem, it suffices to show that, in this circumstance, $u$ is the product of positive matrices.

We do this by induction on the size $n$ of $u$. So suppose that all unitary matrices of determinant 1 and size less than $n$ are products of positive matrices. Without loss of generality we may suppose that $u$ is diagonal, say $u=\operatorname{diag}\left(\lambda_{1}, \ldots, \lambda_{n}\right)$. Then $u=\operatorname{diag}\left(\lambda_{1}, \bar{\lambda}_{1}, 1, \ldots, 1\right) \operatorname{diag}\left(1, \lambda_{1} \lambda_{2}, \lambda_{3}, \ldots, \lambda_{n}\right)$. The first of these factors is a product of positive matrices, by Lemma 1 . The second is of the form $1 \oplus v$, where $v$ is a unitary matrix of determinant 1 , whose size is $n-1$. By the inductive hypothesis, $v$ is the product of positive matrices and therefore, so is $1 \oplus v$. Hence, $u$ is the product of positive matrices, completing the induction.

\section{REFERENCES}

1. C. S. Ballantine, Products of positive definite matrices IV, Linear Alg. Appl. 3 (1970), 79-114. MR 41:1766

2. M. Khalkali, C. Laurie, B. Mathes and H. Radjavi, Approximation by products of positive operators, J. Operator Theory 29 (1993), 237-247. MR 96c:47028

3. G. J. Murphy and N. C. Phillips, $C^{*}$-algebras with the approximate positive factorization property, Trans. Amer. Math. Soc. 348 (1996), 2291-2306. CMP 96:10

4. N. C. Phillips, Every invertible Hilbert-space operator is a product of seven positive operators, Canad. Math. Bull. 38 (1995), 230-236. MR 96h:47044

5. H. Radjavi, Products of Hermitian matrices and symmetries, Proc. Amer. Math. Soc. 21 (1969), 369-372; 26 (1970), 701. MR 39:1470; MR 42:289

6. P. Y. Wu, Products of normal operators, Canadian J. Math. 40 (1988), 1322-1330. MR 90d:47039

Department of Mathematics, University College, Cork, Ireland

E-mail address: gjm@ucc.ie 